\title{
Reconsidering the quantisation of electrodynamics with boundary conditions and some measurable consequences
}

\author{
M. BORDAG ${ }^{1}$ \\ University of Leipzig, Institute for Theoretical Physics \\ Augustusplatz 10/11, 04109 Leipzig, Germany
}

\begin{abstract}
We show that the commonly known conductor boundary conditions $E_{\|}=B_{\perp}=0$ can be realized in two ways which we call 'thick' and 'thin' conductor. The 'thick' conductor is the commonly known approach and includes a Neumann condition on the normal component $E_{\perp}$ of the electric field whereas for a 'thin' conductor $E_{\perp}$ remains without boundary condition. Both types describe different physics already on the classical level where a 'thin' conductor allows for an interaction between the normal components of currents on both sides. On quantum level different forces between a conductor and a single electron or a neutral atom result. For instance, the Casimir-Polder force for a 'thin' conductor is by about $13 \%$ smaller than for a 'thick' one.
\end{abstract}

\section{Introduction}

The quantisation of the electromagnetic field in the presence of conducting boundaries is on the base of a broad area of physical phenomena including the Casimir effect, the retarded interaction of atomic systems with a conducting wall, their radiation properties in a cavity and more which are frequently called Cavity $Q E D$. Many of these phenomena are by now measured with high precision as for example the Casimir effect and play an increasing role in applications like nano-technology.

The interaction with a conducting boundary can be described by the well known boundary conditions

$$
E_{\|}=H_{\perp}=0
$$

which follow from the mobility of the electrons in the boundary surface $S$. The present paper is based on the observation that the conditions (11) leave room for

\footnotetext{
${ }^{1}$ e-mail: Michael.Bordag@itp.uni-leipzig.de
} 
different boundary conditions on the normal component $\mathcal{E}_{\perp}$ of the electric field and, as a consequence, for different physics.

In the commonly adopted approach it is always assumed that $\mathcal{E}_{\perp}$ satisfies a Neumann boundary condition on $S$. In Coulomb gauge this follows after imposing a Dirichlet condition on the electrostatic potential and corresponding conditions on the transverse modes. This procedure was probably first used in [1] to calculate the force acting on a neutral atom in front of a conducting wall (Casimir-Polder force) and in 2 deriving the Casimir effect.

Motivated by the calculation of radiative corrections to the Casimir force and in order to have Lorentz invariance broken only by the boundary in [3] a quantisation of electrodynamics had been developed where the conditions (11) are implemented in a 'minimal' manner. The point is that for the quantisation the use of the electromagnetic potential is indispensable and the boundary conditions on the components of the electromagnetic potential do not follow uniquely from (11). In [3] the path integral formulation in covariant gauge using a restriction of the functional integration space by delta functions on the boundary surface such that the potentials obey (10) had been used. A photon propagator ${ }^{S} D_{\mu \nu}(x, y)$ was derived satisfying (11) and being defined in the hole space, i.e., on both sides of the conducting surface which was assumed to be infinitely thin. This propagator allowed for an easy calculation of the radiative correction in [3] for parallel planes and in [4] for a sphere.

Below we show that the difference between both approaches can be traced back to the boundary conditions on the normal component of the electric field $\mathcal{E}_{\perp}$. Whereas $\mathcal{E}_{\perp}$ fulfils a Neumann conditions in the standard approach it remains without any condition in the approach of [3]. Both approaches describe different physics which can be best described as a 'thick' conductor versus a 'thin' one. For the 'thick' conductor one needs to assume that inside there are electrons which can move freely in the perpendicular direction making the electric field vanish. Then from Gauss law on the surface only a Neumann condition is possible for consistency reasons. In opposite, in the 'thin' conductor one needs to assume that there is no freedom for the electrons to move in the perpendicular direction either due to the thinness or due to an anisotropic conductivity so that there is no need to impose a condition on the normal component of the electric field.

In terms of the electromagnetic potential, for a 'thick' conductor we have boundary conditions on all components of the potential, separated spectral problems on both sides and absolutely no interaction across the conductor. For a 'thin' conductor in the approach of [3] which was in [5] rewritten in terms of new polarisations we have two polarisations with boundary conditions and two without. The latter do not feel the boundary and are the same as in free space. Below we show that from these polarisations a classical force follows between charges on different sides of the surface in case both are moving in the normal direction. This makes the two setups describe different physics.

The question appears about further measurable quantities which are sensitive 
to the difference between both approaches. In QED the first candidate is the Casimir effect. However, in 3 and in subsequent papers the Casimir effect turned out to be the same. The next candidate is the Casimir-Polder force. Below we show that it is for a 'thin' conductor by $13 \%$ smaller than for a 'thick' one.

We note that differences between 'thin' and 'thick' conductors had been reported earlier in [6] and [7] for the fluctuations of the Casimir force between two plates where the difference was in a mode propagating parallel to the plates.

We should stress that no such choice appears if the boundary is considered in the mathematical sense, i.e. when there is no meaning of the space beyond the boundary. Then one has to impose boundary conditions on all components of the electromagnetic potential. As was noticed in the context of quantum cosmology just restricting the fields which satisfy the Coulomb gauge condition may lead to an incorrect result (see, [8, 9]), 10]). In [10] general criteria had been formulated for the compatibility of gauge and boundary conditions.

In the next section we discuss in detail the two sets of boundary conditions, the corresponding mode expansions and propagators. In the third section we calculate the Casimir-Polder force for a 'thin' conductor. Discussions and conclusions are given in the fourth section. In the appendix we display some sum rules used in the text. Throughout the paper we use units with $\hbar=c=1$.

\section{Quantisation with boundary conditions}

In this section we consider the quantisation of electrodynamics with conductor boundary conditions which was introduced in [3. The starting point is the path integral representation,

$$
Z(j)=\int D A_{\mu} \prod_{x \in S, \nu} \delta\left(n^{\mu} F_{\mu \nu}^{*}\right) e^{i S(A)+i A_{\mu} j^{\mu}}
$$

with delta functions on the surface $S$ restricting the integration space in the functional integral to such potentials $A_{\mu}$ that the corresponding field strengths satisfy (1). Here the potentials $A_{\mu}(x)$ are defined in the whole space, i.e., on both sides of the boundary surface $S$. The action $S(A)$ contains the gauge breaking term $1 /(2 \alpha)\left(\partial_{\mu} A^{\mu}\right)^{2}$. The delta function in (2) can be represented as a path integral, $\prod_{x \in S} \delta\left(n^{\mu} F_{\mu \nu}^{*}\right)=\int D b \exp \left(i \int_{S} d x b^{\nu}(x) n^{\mu} F_{\mu \nu}^{*}(x)\right)$, over auxiliary fields $b^{\nu}(x)$ living on the surface $S$ after what the integration over $A_{\mu}$ is Gaussian and results in a new photon propagator ${ }^{S} D_{\mu \nu}(x, y)$, see Eq. (16) below, whose boundary dependent part is quite different from the standard approach. Although this had not yet been worked out in detail, the procedure should be equivalent to a quantisation with constraints where the auxiliary fields $b^{\nu}(x)$ play the role of Lagrange multipliers. 
This propagator although derived in the framework of quantum field theory can be used for the classical problem to find the field of a given source. The Maxwell equation $\partial^{\mu} F_{\mu \nu}=j_{\nu}$ is solved by

$$
A_{\mu}(x)=\int d y{ }^{S} D_{\mu \nu}(x, y) j_{\nu}(y)
$$

and the field strengths which follow from (3) obviously fulfil (10) and do not depend on the gauge in which ${ }^{S} D_{\mu \nu}(x, y)$ was calculated if current conservation $\partial^{\mu} j_{\mu}=0$ holds.

In order to make the discussion more transparent we turn to the completely explicit expressions which can be written down for a single plane surface $S$. We assume it to be located in the point $x^{3} \equiv z=0$. We work in Lorentz gauge with gauge parameter $\alpha=1$ and use the polarisations $E_{\mu}^{s}$ which were introduced in [5] and the mode expansion

$$
A_{\mu}(x)=\int d k_{\|} \int d k_{3} \frac{1}{\sqrt{2 k_{0}}} \frac{e^{i k_{\alpha} x^{\alpha}}}{2 \pi} \sum_{s=0}^{3} E_{\mu}^{s} f_{s}\left(z, k_{3}\right) a_{s, k}+\text { c.c. } .
$$

Here the index $\alpha$ takes values $\alpha=0,1,2$ and c.c. indicates that the complex conjugated expression must be added. The $a_{s, k}$ are the free coefficients in the classical expression which become creation and annihilation operators when doing quantisation. The mode functions $f_{s}\left(z, k_{3}\right)$ are discussed below. The frequency is $k_{0}=\sqrt{k_{\|}^{2}+k_{3}^{2}}$ where $k_{\|} \equiv \sqrt{k_{1}^{2}+k_{2}^{2}}$ is the momentum parallel to the plane. In this representation the polarisations read

$$
E_{\mu}^{0}=\frac{1}{\Gamma}\left(\begin{array}{c}
k_{0} \\
k_{1} \\
k_{2} \\
0
\end{array}\right), E_{\mu}^{3}=\left(\begin{array}{c}
0 \\
0 \\
0 \\
1
\end{array}\right), E_{\mu}^{1}=\frac{1}{k_{\|}}\left(\begin{array}{c}
0 \\
k_{2} \\
-k_{1} \\
0
\end{array}\right), E_{\mu}^{2}=\frac{1}{\Gamma k_{\|}}\left(\begin{array}{c}
k_{\|}^{2} \\
k_{0} k_{1} \\
k_{0} k_{2} \\
0
\end{array}\right)
$$

with $\Gamma=\sqrt{k_{\alpha} k^{\alpha}} \equiv \sqrt{k_{0}^{2}-k_{\|}^{2}}$. They form a basis,

$$
g_{\mu \nu}=E_{\mu}^{s} g_{s t} E_{\nu}^{t}
$$

where $g_{s t}=\operatorname{diag}(1,-1,-1,-1)$ is a metric tensor like $g_{\mu \nu}$. The third polarisation, $E_{\mu}^{3}$, is the normal vector to $S$ and the polarisations with $s=1,2$ are transversal, $k^{\mu} E_{\mu}^{s=1,2}=0$. In order to apply the boundary conditions (11) to (4) we remark that they can be written in terms of the dual field strength and take the form $n^{\mu} F_{\mu \nu}^{*}=2 \epsilon_{\mu \nu \lambda \delta} n^{\mu} \partial^{\lambda} A^{\delta}$. Inserting the mode expansion (4) it is obvious that the polarisations with $s=0,3$ drop out and conditions only on the mode functions $f_{s=1,2}\left(z, k_{3}\right)$ follow. Because the boundary conditions and the polarisations do not contain derivatives with respect to $z$ these are Dirichlet conditions, i.e.,

$$
f_{s=1,2}\left(z, k_{3}\right)=0 \quad \text { for } \quad z=0 .
$$


Then the corresponding mode functions are

$$
f_{s=1,2}\left(z, k_{3}\right)=\frac{\sin \left(k_{3} z\right)}{\sqrt{\pi / 2}} .
$$

For the polarisations with $s=0,3$ we do not have boundary conditions, hence they take the same form as in free space,

$$
f_{s=0,3}\left(z, k_{3}\right)=\frac{e^{i k_{3} z}}{\sqrt{2 \pi}} .
$$

In the mode expansion (44) the integration over $k_{\|}$goes over the whole $\mathbb{R}^{2}$. For the polarisations $s=0,3$ the integration over $k_{3}$ goes also over the whole axis, $k_{3} \in(-\infty, \infty)$, but for $s=1,2$ only over the half axis, $k \in[0, \infty)$. Because of the boundary conditions, for $s=1,2$ we have two completely separated problems on both sides of the surface and, strictly, speaking, we should introduce independent constants according to $a_{s, k} \rightarrow \Theta(-z) a_{-, s, k}+\Theta(z) a_{+, s, k}$. The mode expansion (44) has the property to solve the Maxwell equations in the gauge $\alpha=1$ and to fulfil the boundary conditions (11).

Next we consider the corresponding electric and magnetic fields strengths. By means of $\mathcal{E}=\frac{\partial}{\partial x^{0}} A_{i}-\frac{\partial}{\partial x^{2}} A_{0}$ we obtain from (44)

$$
\begin{aligned}
\mathcal{E}_{i}= & \int d k_{\|} \int d k_{3} \frac{1}{\sqrt{2 k_{0}}} \frac{e^{i k_{\alpha} x^{\alpha}}}{2 \pi}\left\{\left(\begin{array}{l}
0 \\
0 \\
1
\end{array}\right)_{i} i k_{0}\left(-a_{0, k}+a_{3, k}\right) \frac{e^{i k_{3} z}}{\sqrt{2 \pi}}\right. \\
& \left.+\left(\begin{array}{c}
k_{2} \\
-k_{1} \\
0
\end{array}\right)_{i} \frac{i k_{0}}{k_{\|}} \frac{\sin \left(k_{3} z\right)}{\sqrt{\pi / 2}} a_{1, k}+\left(\begin{array}{c}
k_{1} k_{3} \sin \left(k_{3} z\right) \\
k_{2} k_{3} \sin \left(k_{3} z\right) \\
i k_{\|}^{2} \cos \left(k_{3} z\right)
\end{array}\right)_{i} \frac{i a_{2, k}}{k_{\|} \sqrt{2 \pi}}\right\}
\end{aligned}
$$

and from $B_{i}=\epsilon_{i j k} \frac{\partial}{\partial x^{j}} A_{k}$ for the magnetic field

$$
\begin{aligned}
B_{i}= & \int d k_{\|} \int d k_{3} \frac{1}{\sqrt{2 k_{0}}} \frac{e^{i k_{\alpha} x^{\alpha}}}{2 \pi}\left\{( \begin{array} { c } 
{ k _ { 2 } } \\
{ - k _ { 1 } } \\
{ 0 }
\end{array} ) _ { i } i \left(-a_{0, k}+a_{3, k} \frac{e^{i k_{3} z}}{\sqrt{2 \pi}}\right.\right. \\
& \left.+\left(\begin{array}{c}
k_{1} k_{3} \cos \left(k_{3} z\right) \\
k_{2} k_{3} \cos \left(k_{3} z\right) \\
-i k_{\|}^{2} \sin \left(k_{3} z\right)
\end{array}\right)_{i} \frac{a_{1, k}}{k_{\|} \sqrt{2 \pi}}+\left(\begin{array}{c}
k_{2} \\
-k_{1} \\
0
\end{array}\right)_{i} \frac{-k_{0}}{k_{\|}} \frac{\cos \left(k_{3} z\right)}{\sqrt{\pi / 2}} a_{2, k}\right\}
\end{aligned}
$$

follows. It is seen that the normal component of the electric field has a contribution which does not obey a boundary condition although the boundary conditions (11) are obviously fulfilled. The same holds for the parallel components of the magnetic field. 
The connection with the commonly used formulation can be established by applying a Neumann condition to the normal component of the electric field,

$$
\frac{\partial}{\partial z} \mathcal{E}_{3}=0 \text { for } \quad z=0
$$

For this to hold we must take

$$
f_{0}\left(z, k_{3}\right)=i \frac{\sin \left(k_{3} z\right)}{\sqrt{\pi / 2}}, \quad f_{3}\left(z, k_{3}\right)=\frac{\cos \left(k_{3} z\right)}{\sqrt{\pi / 2}}
$$

in the mode expansion (41) instead of (91) together with the restriction of the integration $k_{3} \in[0, \infty)$. After that, by means of completeness of the polarisations $E_{\mu}^{s}$, (5), the mode expansion (4) must be equivalent to the standard one which can be written in the form

$$
\begin{aligned}
& A_{\mu}(x)=\int d k_{\|} \int_{0}^{\infty} d k_{3} \frac{1}{\sqrt{2 k_{0}}} \frac{e^{i k_{\alpha} x^{\alpha}}}{2 \pi} \\
& \times\left\{\left(\begin{array}{c}
1 \\
0 \\
0 \\
0
\end{array}\right) \frac{\sin \left(k_{3} z\right)}{\sqrt{\pi / 2}} a_{0, k}+\left(\begin{array}{c}
0 \\
k_{1} \sin \left(k_{3} z\right) \\
k_{2} \sin \left(k_{3} z\right) \\
-i k_{3} \cos \left(k_{3} z\right)
\end{array}\right) \frac{a_{3, k}}{k \sqrt{\pi / 2}}\right. \\
& \left.+\left(\begin{array}{c}
0 \\
k_{2} \\
-k_{1} \\
0
\end{array}\right) \frac{\sin \left(k_{3} z\right)}{k_{\|} \sqrt{\pi / 2}} a_{1, k}+\left(\begin{array}{c}
k_{1} k_{3} \sin \left(k_{3} z\right) \\
k_{2} k_{3} \sin \left(k_{3} z\right) \\
i k_{\|}^{2} \cos \left(k_{3} z\right)
\end{array}\right) \frac{a_{2, k}}{k k_{\| \sqrt{\pi / 2}}}\right\}+ \text { c.c. }
\end{aligned}
$$

with $k=\sqrt{k_{\|}^{2}+k_{3}^{2}}$. The equivalence is established by the relations

$$
a_{2}-\tilde{a}_{2}=i \frac{k_{\|}}{k}\left(\tilde{a}_{0}-\tilde{a}_{3}\right)=-\frac{k_{\|}}{k_{3}}\left(a_{0}-a_{3}\right)
$$

where the $a_{s}$ belonging to the mode expansion (44) are marked by a tilde. All quantities which are calculated either in the standard approach with the mode expansion (14) or with the 'new' polarisations $E_{\mu}^{s}$, (15), and the additional condition (12) on the normal component of the electric field, must deliver the same physical results. As we will discuss in the next section, they do indeed. However, if we relax that condition on $\mathcal{E}_{\perp}$ they will not. In this way we established that the mode expansion (4) with the mode functions (8) and (13) (instead of (9)) is equivalent to the commonly used one so that the whole difference is in the boundary condition (12) on the normal component of the electric field.

In the remaining part of this section we collect some formulas which we need for the calculation of the Casimir-Polder force in the next section. The photon propagator ${ }^{S} D_{\mu \nu}(x, y)$ was derived in [3] in general form and explicitly for two 
parallel planes. Here we restrict ourselves to the easier case of one plane. The propagator takes the form

$$
{ }^{S} D_{\mu \nu}(x, y)=D_{\mu \nu}(x-y)+\bar{D}_{\mu \nu}(x, y),
$$

where

$$
D_{\mu \nu}(x-y)=\int \frac{d^{4} k}{(2 \pi)^{4}} \frac{e^{i k_{\mu}\left(x^{\mu}-y^{\mu}\right)}}{-k_{0}^{2}+\vec{k}^{2}-i \epsilon}\left(g_{\mu \nu}-(1-\alpha) \frac{k_{\mu} k_{\nu}}{k^{2}}\right)
$$

is the (causal if $\epsilon>0$ ) photon propagator in free space with a gauge parameter $\alpha$ and $\bar{D}_{\mu \nu}(x, y)$ is the boundary dependent part,

$$
\bar{D}_{\mu \nu}(x, y)=\int \frac{d^{3} k_{\alpha}}{(2 \pi)^{3}} \frac{e^{i k_{\alpha}\left(x^{\alpha}-y^{\alpha}\right)+i \Gamma\left(\left|x^{3}\right|+\left|y^{3}\right|\right)}}{-2 i \Gamma} \sum_{s=1,2} E_{\mu}^{s} E_{\nu}^{s},
$$

with $\alpha=0,1,2$ and the polarisation vectors $E_{\mu}^{s}$ given by (5).

Representation (18) can be derived in the following way. Consider the scalar propagator in free space (17) without the Lorentz structure and integrate over $k_{3}$,

$$
D(x-y)=\int \frac{d^{4} k}{(2 \pi)^{4}} \frac{e^{i k_{\mu}\left(x^{\mu}-y^{\mu}\right)}}{-k_{0}^{2}+\vec{k}^{2}-i \epsilon}=\int \frac{d^{3} k_{\alpha}}{(2 \pi)^{3}} \frac{e^{i k_{\alpha}\left(x^{\alpha}-y^{\alpha}\right)+i \Gamma\left|x^{3}-y^{3}\right|}}{-2 i \Gamma} .
$$

Next observe that the boundary conditions (11) in terms of the new polarisations are two Dirichlet conditions. The corresponding scalar propagator can be expressed in the form

$$
D^{\text {Dirichlet }}(x, y)=\int \frac{d^{3} k_{\alpha}}{(2 \pi)^{3}} \frac{e^{i k_{\alpha}\left(x^{\alpha}-y^{\alpha}\right)}}{-2 i \Gamma}\left(e^{i \Gamma\left|x^{3}-y^{3}\right|}-e^{i \Gamma\left(\left|x^{3}\right|+\left|y^{3}\right|\right)}\right) .
$$

It is obvious that it fulfils the boundary condition in $x^{3}=0$ or in $y^{3}=0$. For both arguments on one side the second contribution is that of a mirror charge. For these arguments on different sides, for example $x^{3}>0$ and $y^{3}<0$, it is zero.

Finally, $\bar{D}_{\mu \nu}(x, y)$, (18), follows from the second part in $D^{\text {Dirichlet }}(x, y)$, (20), as the projection on the two polarisations which are affected by the boundary conditions by inserting the corresponding polarisation vectors.

Let us note also for later use that $D^{\text {Dirichlet }}(x, y)$, (20) , can be obtained from the more conventional representation

$$
D^{\text {Dirichlet }}(x, y)=\int \frac{d k_{\alpha}}{(2 \pi)^{3}} \int_{0}^{\infty} \frac{d k_{3}}{\pi / 2} \frac{e^{i k_{\alpha}\left(x^{\alpha}-y^{\alpha}\right)} \sin \left(k_{3} x^{3}\right) \sin \left(k_{3} y^{3}\right)}{-k_{0}^{2}+\vec{k}^{2}-i \epsilon}
$$


by integrating over $k_{3}$. Another well known representation emerges if in (21) integrating over $k_{0}$,

$$
D^{\text {Dirichlet }}(x, y)=i \int \frac{d^{2} k_{\|}}{(2 \pi)^{2}} \int_{0}^{\infty} \frac{d k_{3}}{\pi / 2} \frac{1}{2 k_{0}} e^{-i k_{\alpha}\left(x_{\alpha}-y_{\alpha}\right)} \sin \left(k_{3} x^{3}\right) \sin \left(k_{3} y^{3}\right)
$$

with $k_{0}=\sqrt{k_{\|}^{2}+k_{3}^{2}}$ which appears in this way written in terms of the mode functions (8). Note that in opposite to (20) the latter two representations are valid only if both arguments are on one side of the boundary surface. We conclude by writing the propagator (16) in the form of (22). Taking $\alpha=1$ and assuming $x^{3}$ and $y^{3}$ to be on one side of the surface it reads

$$
\begin{aligned}
{ }^{S} D_{\mu \nu}(x, y)= & \int \frac{d k_{\alpha}}{(2 \pi)^{3}} e^{i k_{\alpha}\left(x^{\alpha}-y^{\alpha}\right)} \\
& \times\left\{\int_{-\infty}^{\infty} \frac{d k_{3}}{2 \pi} \frac{e^{i k_{3}\left(x^{3}-y^{3}\right)}}{-k_{0}^{2}+k_{\|}^{2}+k_{3}^{2}-i \epsilon} \sum_{s, t=0,3} E_{\mu}^{s} g_{s t} E_{\nu}^{t}\right. \\
& \left.+\int_{0}^{\infty} \frac{d k_{3}}{\pi / 2} \frac{\sin \left(k_{3} x^{3}\right) \sin \left(k_{3} y^{3}\right)}{-k_{0}^{2}+k_{\|}^{2}+k_{3}^{2}-i \epsilon} \sum_{s, t=1,2} E_{\mu}^{s} g_{s t} E_{\nu}^{t}\right\} .
\end{aligned}
$$

This propagator consists of two parts. The first one involving the polarisations $s=0,3$ is the same as in free space and it is defined in the hole space and the second one involving the polarisations $s=1,2$ is defined for both arguments on one side of the boundary. For arguments on different sides it must be dropped.

For the boundary conditions of a 'thick' conductor a representation of the propagator in parallel to (16) can be written down too. It starts from the observation that the polarisation $s=0$ according to (13) goes with a Dirichlet condition and using (20) we can simply include the second contribution into $\bar{D}_{\mu \nu}(x, y)$ (18). For the other polarisation, $s=3$, we have from (13) a Neumann condition. The corresponding scalar propagator is given by Eq. (20) with a plus sign between the two exponentials. In this way we can include it into $\bar{D}_{\mu \nu}(x, y)$ (18) in the same way as before only with a reversed sign. Summarising we obtain the photon propagator for the 'thick' boundary conditions for $\alpha=1$ in the form of ${ }^{S} D_{\mu \nu}(x, y)$, (16), with a boundary dependent part which is now given by

$$
\bar{D}_{\mu \nu}(x, y)=\int \frac{d^{3} k_{\alpha}}{(2 \pi)^{3}} \frac{e^{i k_{\alpha}\left(x^{\alpha}-y^{\alpha}\right)+i \Gamma\left(\left|x^{3}\right|+\left|y^{3}\right|\right)}}{-2 i \Gamma}\left(-E_{\mu}^{0} E_{\nu}^{0}-E_{\mu}^{3} E_{\nu}^{3}+E_{\mu}^{1} E_{\nu}^{1}+E_{\mu}^{2} E_{\nu}^{2}\right) \text {. }
$$

We wrote the sum over the polarisations explicitly to underline the reversed sign for $s=3$ which is due to the Neumann condition for this polarization. From the above derivation it follows that ${ }^{S} D_{\mu \nu}(x, y)$ with this $\bar{D}_{\mu \nu}(x, y)$ is just another 
representation of the standard photon propagator which follows from the mode expansion (14).

In order to further discuss the physical meaning of the 'thin' conductor we use the representation (23) of the propagator to calculate the interaction between two currents, $j_{1}(x)$ and $j_{2}(y)$, which may be on the same or on different sides of the boundary. According to (3) it is given by

$$
\int j_{\mu}(z){ }^{S} D_{\mu \nu}\left(z, z^{\prime}\right) j_{\nu}\left(z^{\prime}\right) d z d z^{\prime}
$$

For charges at different sides only the contributions from the polarisations $s=0,3$ are nonzero. First we consider the charges to be static. Then the corresponding current has only a $(\mu=0)$-component and that is independent on time, i.e., on $z_{0}$. Hence the integration over $z_{0}$ can be carried out delivering in (23) a delta function $\delta\left(k_{0}\right)$. This gives as usual the Greens function known from electrostatics. But the only remaining $(\mu=0)$-component is that in $E_{\mu}^{0}$ which is proportional to $k_{0}$ so that the whole expression (25) vanishes. in this way a charge at rest does not interact with a current on the other side. For reasons of Lorentz invariance the same holds for a charge moving in parallel to the plane. The situation changes only if both charges are moving in perpendicular direction. In that case the interaction (25) between them across the surface is nonzero. This must be considered as a difference in physics between a 'thin' and a 'thick' conductor on the classical level. In the next section we show that this is the case on the quantum level too.

The boundary conditions of a 'thin' conductor may seem strange from the point of view of the commonly known ones. We would like to stress here that they can be used in a consistent way to derive either a mode expansion or the corresponding photon propagator as the above discussions have shown. They are based on the assumption that the conductor is infinitely thin, which of course cannot be realized in nature. The question whether it can be a good approximation to some physical situation can be answered only by investigation of interactions like (25) above.

The Casimir effect as the force acting between conducting surfaces deserves a special consideration with respect to the two sets of boundary conditions because it is the same for both. This was in fact shown in [3] where a 'thin' conductor was used and the standard result for the Casimir force was re-obtained. Later in [4] the same had been shown for a sphere. The point is that the Casimir effect is quite insensitive because it makes use only of the fact that two boundary dependent polarisations $\left(E_{\mu}^{s}\right.$ with $\left.s=1,2\right)$ are present. In other words it counts the degrees of freedom in opposite to the Casimir-Polder force where the time-component plays a special role.

At this place we should remark that in the usual approach in Coulomb gauge the Casimir force receives two contributions from the two transversal photons which correspond to the polarisations $s=1,2$ in (14). If using Lorentz gauge 
with the same polarisations there are four contributions obeying boundary conditions, namely in addition the time-like and the longitudinal photon $(s=0,3$ in (14)). The result should be a doubled Casimir force. As it was shown in 11] by demanding BRST invariance the ghosts become boundary dependent too and give two compensating contributions returning the Casimir force to its previous value. Although the ghosts do not couple to physical fields we have to stress that they may be important in other physical situations (see [12] and [13] for a discussion regarding ghost fields in dielectrics).

The situation with the time-like and the longitudinal photons is different when calculating the Casimir-Polder force. Here there are no contributions from the ghosts because they do not couple to the electron and it can be shown that if performing the calculation in Lorentz gauge using the mode expansion (14) the contributions form the modes with $s=0$ and $s=3$ compensate each other.

Another moment to be mentioned is the relation to the force between two dielectric bodies or between a charge and a dielectric body. In that case there is automatically a condition on the normal component of the electric field present $\left(\epsilon \mathcal{E}_{z}\right.$ must be continuous) so that this case turns in the limit into a 'thick' conductor.

\section{Interaction of a charge with a conducting boun- dary}

In this section we calculate the force acting on a single electron or on an atom in front of a conducting boundary for a 'thin' conductor, i.e., without imposing a boundary condition on the normal component of the electric field. The case of a 'thick' conductor, i.e., with the Neumann condition on the normal component of the electric field, is matter of the standard calculation using the mode expansion (14) and can be found in numerous papers. Besides the original one [1] we mention [14 and successors, [15], [16] and the book [17. Here we show that the same result can be obtained in terms of the polarisations $E_{\mu}^{s}$, Eq. (15), with the mode functions (13) or, equivalently, with the photon propagator (24).

The calculation for a 'thin' conductor starts from the non relativistic Hamilton operator

$$
H=\frac{(\vec{p}-e \vec{A})^{2}}{2 m}+e A_{0}+V(x),
$$

for a particle in some potential $V(r)$ which is coupled to the electromagnetic field $A_{\mu}$. Note that we have to keep $A_{0}$ because we are not working in Coulomb gauge. We divide $H$ into an unperturbed part,

$$
H_{0}=\frac{p^{2}}{2 m}+V(x)
$$


and a perturbation. For the unperturbed problem,

$$
H_{0} \psi_{n}(x)=E_{n} \psi_{n}(x)
$$

it is assumed that the $\psi_{n}(x) \equiv \mid n>$ form a basis and that the expectation values $<n\left|p_{i}\right| n^{\prime}>$ are finite. The wave function is assumed to be centred in a point at distance $a$ from the surface. To complete the setup one has to enlarge the state space of the $\psi_{n}$ 's by the photon states.

For a single electron in front of a conducting wall the potential $V(r)$ must be non electrostatic (a magnetic field in a Penning trap for instance). It is needed in order to keep the electron away from a direct contact with the surface.

For an atom in front of the conducting wall the potential $V(r)$ is that of the nucleus,

$$
V(x)=\frac{-e^{2}}{4 \pi\left|x-x_{N}\right|},
$$

which is at the position $x_{N}=(0,0, a)$, i.e., at distance $a$ from the surface.

The perturbation is given by the operator

$$
\Delta H=-\frac{e}{m} \vec{A} \vec{p}-\frac{e}{2 m}(\vec{p} \vec{A})+e A_{0}+\frac{e^{2}}{2 m} \vec{A}^{2}+\Delta V(x)
$$

where in the second term in the r.h.s. $\vec{p}=-i \nabla$ acts on $\vec{A}(x)$. We have to keep this term when not working in Coulomb gauge. For the setup with an atom

$$
\Delta V(x)=\frac{e^{2}}{4 \pi \sqrt{x^{2}+y^{2}+(z+2 a)^{2}}}
$$

is the contribution of the mirror charge of the nucleus. It appears as an external field whereas the mirror charge of the electron (taken alone or in the atom) comes in from the interaction with the electromagnetic field in $\Delta H$. For a single charge $\Delta V$ is not present.

The perturbations are calculated according to the well known rules of quantum mechanics. From $\Delta V(x)$ we have a first order perturbation simply by taking it in the unperturbed state,

$$
\delta_{\mathrm{es}, \mathrm{nucl}}=\frac{e^{2}}{4 \pi}\left(\frac{1}{2 a}+\frac{Q}{16 a^{3}}+\ldots\right),
$$

with $Q=<n\left|2 x_{3}^{2}-x_{1}^{2}-x_{2}^{2}\right| n>$. Also the next-to-last term gives rise the a first order perturbation,

$$
\delta_{1}=\frac{e^{2}}{2 m}<n, 0_{\gamma}\left|\vec{A}^{2}\right| n, 0_{\gamma}>,
$$


where $\mid 0_{\gamma}>$ denotes the photon ground state, whereas the other ones contribute in second order according to the well known formula

$$
\Delta E_{n}^{(2)}=\sum_{n^{\prime}} \sum_{s, k} \frac{\left|<n, 0_{\gamma}\right| \Delta H^{(2)}\left|n^{\prime},(s, k)_{\gamma}>\right|^{2}}{E_{n}-\left(E_{n^{\prime}}+\omega\right)}
$$

where $\Delta H^{(2)}$ is that part in $\Delta H,(\underline{30})$, which is linear in $A_{\mu}$. Here the intermediate states $\mid n^{\prime},(s, k)_{\gamma}>$ include all one-photon states.

The electromagnetic potentials are given by Eq. (4) where the $a_{s, k}$ and their complex conjugate are now annihilation and creation operators. The polarisations are given by (5) and the mode functions by (8) for $s=1,2$ and in dependence on the choice of 'thick' or 'thin' boundary conditions by the mode functions (13) resp. (9) for $s=0,3$.

In order to calculate the expectation value in the first order contribution $\delta_{1}$, (33), we need the mode expansion (44) and keep only the boundary dependent contributions, i.e., the polarisations $s=1,2$. The vacuum expectation value takes the form

$$
<0_{\gamma}\left|A_{\mu}(x) A_{\nu}(y)\right| 0_{\gamma}>=\int d^{2} k_{\|} \int_{0}^{\infty} d k_{3} \frac{1}{\sqrt{2 k_{0}}} \sum_{s=1,2} E_{\mu}^{s} E_{\nu}^{s} f_{s}\left(x, k_{3}\right) f_{s}^{*}\left(y, k_{3}\right) .
$$

Using (22), (201) and (18) it can be expressed in terms of the boundary dependent part of the propagator,

$$
\delta_{1}=\frac{e^{2}}{2 m} \frac{1}{i}<n\left|\bar{D}_{i i}(x, x)\right| n>
$$

where the boundary independent part was dropped. Using the explicit form, (18), and $\left|x^{3}\right|=x^{3}$ because we use $x^{3}>0$ only, we obtain

$$
\delta_{1}=\frac{e^{2}}{2 m} \frac{1}{i} \int \frac{d^{3} k_{\alpha}}{(2 \pi)^{3}} \frac{-e^{2 i \Gamma a}}{-2 i \Gamma}\left(1+\frac{k_{0}^{2}}{\Gamma^{2}}\right)<n\left|e^{2 i \Gamma\left(x^{3}-a\right)}\right| n>.
$$

Here the factor $k_{0}^{2} / \Gamma^{2}$ comes from the second polarisation $(s=2)$. After a Wick rotation, $k_{0} \rightarrow i k_{4}, \Gamma \rightarrow i \gamma \equiv i \sqrt{k_{4}^{2}+k_{\|}^{2}}$ and expanding the exponential, $\exp \left(2 i \Gamma\left(x^{3}-a\right)\right)=1+\ldots$ (the electron wave function is centred in $\left.x^{3}=a\right)$,

$$
\begin{aligned}
\delta_{1} & \left.=-\frac{e^{2}}{2 m} \int \frac{d_{\mathrm{E}}^{3} k}{(2 \pi)^{3}} \frac{e^{-2 \gamma a}}{2 \gamma}\left(1+\frac{k_{4}^{2}}{\gamma^{2}}\right)<n \mid 1+\ldots\right) \mid n> \\
& =-\frac{e^{2}}{4 \pi} \frac{1+1 / 3}{8 \pi m a^{2}}+O\left(\frac{1}{a^{4}}\right)
\end{aligned}
$$

follows, where in $(1+1 / 3)$ the ' 1 ' comes from the first polarisation $(s=1)$ (it is the same as in the standard approach) whereas the ' $1 / 3$ ' comes from $s=2$ (it is different). 
The second order perturbation (34) is

$$
\delta_{2}=\sum_{n^{\prime}} \int \frac{d^{2} k_{\|}}{(2 \pi)^{2}} \int_{0}^{\infty} \frac{d k_{3}}{\pi / 2} \sum_{s=1,2} \frac{1}{2 \omega} \frac{|<n| G_{s}\left|n^{\prime}>\right|^{2}}{E_{n}-\left(E_{n^{\prime}}+\omega\right)}
$$

where the sum over $s$ and the integration over $k$ come from the intermediate photon states and the mode expansion (4) together with the usual commutator relation have been used. From the first three terms in the r.h.s. of (30) the matrix elements become

$$
G_{s}=\frac{e^{i k_{t} x^{t}}}{2 \pi} f_{s}\left(x, k_{3}\right)\left(\frac{-e}{m} E_{t}^{s} p_{t}+e E_{0}^{s}\right),
$$

where (4) and the mode functions (8) have been used. Here and in the following the index $t$ takes values $t=1,2$ and summation over repeated $t$ 's is assumed.

Now we wish to use the new propagator $\bar{D}_{\mu \nu}(x, y)$, Eq. (18). For this reason we rewrite (39) by introducing the integration over $k_{0}$,

$$
\delta_{2}=\sum_{n^{\prime}} \int \frac{d^{2} k_{\|}}{(2 \pi)^{2}} \int_{0}^{\infty} \frac{d k_{3}}{\pi / 2} \sum_{s} \int_{-\infty}^{\infty} \frac{d k_{0}}{2 \pi i} \frac{1}{-k_{0}^{2}+\vec{k}^{2}-i \epsilon} \frac{|<n| G_{s}\left|n^{\prime}>\right|^{2}}{-k_{0}+E_{n}-E_{n^{\prime}}(1-i \epsilon)},
$$

where the integration has to pass the poles according to $\epsilon>0$. This is in parallel to the relation between formulas (21) and (22). Note that representation (41) is in this form valid only if no intermediate antiparticle states are present as it is the case in our non relativistic approximation. A completely relativistic description had been given in [18, and in [19] from which [41) can be obtained in principle (it is quite tedious to do that in detail).

We proceed by carrying out the integration over $k_{3}$ in the same way as (201) follows from (21), where in the factors $\sin \left(k_{3} x^{3}\right) \sin \left(k_{3} y^{3}\right)$, which are contained in (21), the variable $x^{3}$ belongs to one of the scalar products $\left\langle n|\cdots| n^{\prime}\right\rangle$ and $y^{3}$ to the other one. Taking only the boundary dependent part we obtain

$$
\delta_{2}=\sum_{n^{\prime}} \sum_{s} \frac{1}{i} \int \frac{d^{3} k_{\alpha}}{(2 \pi)^{3}} \frac{-e^{2 i \Gamma a}}{-2 i \Gamma} \frac{|<n| \tilde{G}_{s}\left|n^{\prime}>\right|^{2}}{-k_{0}+E_{n}-E_{n^{\prime}}(1-i \epsilon)}
$$

with the notation

$$
\tilde{G}_{s}=e^{i k_{t} x^{t}+i \Gamma\left(x^{3}-a\right)}\left(\frac{-e}{m} E_{t}^{s} p_{t}+e E_{0}^{s}\right) .
$$

Now the matrix elements must be calculated. Taking into account simplifications like that following in the integration over $k_{\alpha}$ from the symmetry under rotations 
in the $k_{t}$-plane we obtain

$$
\begin{aligned}
|<n| \tilde{G}_{1}\left|n^{\prime}>\right|^{2}= & \frac{e^{2}}{m^{2}} \frac{1}{2} \sum_{t}|<n| p_{t}\left|n^{\prime}>\right|^{2} \\
|<n| \tilde{G}_{2}\left|n^{\prime}>\right|^{2}= & \frac{e^{2}}{m^{2}} \frac{k_{0}^{2}}{\Gamma^{2}} \frac{1}{2} \sum_{t}|<n| p_{t}\left|n^{\prime}>\right|^{2} \\
& +e^{2} \frac{k_{\|}^{2}}{\Gamma^{2}}|<n| \varphi\left|n^{\prime}>\right|^{2}
\end{aligned}
$$

with

$$
\varphi=e^{i k_{t} x^{t}+i \Gamma\left(x^{3}-a\right)} .
$$

Several dropped cross terms can be shown not to contribute to the results below and in the contributions proportional to $p_{t}$ we have put $\varphi=1$.

We proceed with doing approximations. Let us first consider a single electron in front of the conducting plane. Here one uses the so-called 'no-recoil' approximation which means that $E_{n}-E_{n^{\prime}}$ in the denominator of (42), i.e., in the electron propagator, is small,

$$
\frac{1}{-k_{0}+E_{n}-E_{n^{\prime}}}=-\frac{1}{k_{0}}-\frac{E_{n}-E_{n^{\prime}}}{k_{0}^{2}}-\frac{\left(E_{n}-E_{n^{\prime}}\right)^{2}}{k_{0}^{3}}+\ldots
$$

This corresponds to an expansion in powers of the ratio of the distance $a$ to the transition wave lengths corresponding to the energy levels $E_{n}$. The second term in the r.h.s. can be shown not to contribute in the following for symmetry reasons. The wave function $\varphi$, Eq. (44), will be expanded in powers of the momentum,

$$
\varphi=1+i k_{t} x^{t}+i \Gamma\left(x^{3}-a\right)+\ldots
$$

which corresponds to an expansion in powers of the ratio of the radius of the orbit of the electron motion in the potential $V(x)$ in Eq. (26) to a. Both ratios are assumed to be small. We obtain

$$
\begin{aligned}
& \delta_{2}^{\text {no-recoil }}=\int \frac{d^{3} k_{\alpha}}{(2 \pi)^{3}} \frac{-e^{2 i \Gamma a}}{-2 i \Gamma} \frac{-1}{k_{0}}\left\{-e^{2} \frac{k_{\|}^{2}}{\Gamma^{2}}\right. \\
& +\sum_{n^{\prime}}\left[\frac{e^{2}}{m^{2}}\left(1+\frac{k_{0}^{2}}{\Gamma^{2}}\right) \frac{1}{2} \sum_{t}|<n| p_{t}\left|n^{\prime}>\right|^{2}\right. \\
& \left.\left.+e^{2} \frac{k_{\|}^{2}}{\Gamma^{2}}\left(k_{\|}^{2} \frac{1}{2} \sum_{t}|<n| x^{t}\left|n^{\prime}>\right|^{2}-\Gamma^{2}|<n| x^{3}-a\left|n^{\prime}>\right|^{2}\right)\left(E_{n}-E_{n^{\prime}}\right)^{2}\right]\right\} .
\end{aligned}
$$


Now we use the sum rule (174) and $\Gamma=\sqrt{k_{0}^{2}-k_{\|}^{2}}$ and arrive at

$$
\begin{aligned}
\delta_{2}^{\text {no-recoil }}= & \int \frac{d^{3} k_{\alpha}}{(2 \pi)^{3}} \frac{-e^{2 i \Gamma a}}{-2 i \Gamma} \frac{-1}{k_{0}}\left\{e^{2}\left(-1+\frac{k_{0}^{2}}{\Gamma^{2}}\right)\right. \\
& \left.+\frac{e^{2}}{m^{2}}\left[\left(-1+\frac{2 k_{0}^{2}}{\Gamma^{2}}+\frac{\Gamma^{2}}{k_{0}^{2}}\right) \frac{1}{2} \sum_{t}<p_{t}^{2}>+\left(-1+\frac{\Gamma^{2}}{k_{0}^{2}}\right)<p_{3}^{2}>\right]\right\},
\end{aligned}
$$

where we used $\sum_{n^{\prime}}\left|n^{\prime}><n^{\prime}\right|=1$. The integration over $k_{0}$ can be carried out in the following way. First we note that in order to make the approximation (45) we have to pass the pole in $k_{0}=0$ from below (see Eq. (41)) at some finite distance. Then we are left with the $k_{0}$-integrations

$$
\int_{-\infty}^{\infty} \frac{d k_{0}}{2 \pi} \frac{f\left(k_{0}^{2}\right)}{k_{0}-i \epsilon}=\frac{i}{2} f(0) \text { and } \int_{-\infty}^{\infty} \frac{d k_{0}}{2 \pi} \frac{f\left(k_{0}^{2}\right)}{k_{0}^{3}-i \epsilon}=\frac{i}{2} f^{\prime}(0) .
$$

Hence in Eq. (48) the terms with a factor $k_{0}^{2}$ in the numerator vanish. The terms with a factor $\Gamma^{2} / k_{0}^{2}$ can be shown to vanish after carrying out the integration over $k_{\|}$. In this way we arrive at

$$
\delta_{2 \text { thin }}^{\text {no-recoil }}=\frac{e^{2}}{4 \pi} \frac{1}{4 a}\left(-1-\frac{1}{2 m^{2}} \sum_{t}<p_{t}^{2}>-\frac{1}{m^{2}}<p_{3}^{2}>\right),
$$

where we added the subscript 'thin'. Here the first contribution in the r.h.s. is the pure electrostatic contribution from the mirror charge of the electron, the other two are the corrections in the case of 'thin' boundary conditions.

Now we consider an atom in front of the plane. In $\delta_{2}$, Eq. (42), we want to perform the Wick rotation and assume $\mid n>$ to be the ground state. In rotating the integration path of $k_{0}$ only the pole in $k_{0}=0$ from $E_{n^{\prime}}=E_{n}$ comes close to the integration path ${ }^{2}$. We assume the ground state to be non-degenerated. Than its contribution to the sum over $n^{\prime}$ can be calculated by the well known formula

$$
\int_{-\infty}^{\infty} \frac{d k_{0}}{2 \pi} \frac{f\left(k_{0}\right)}{k_{0}-i \epsilon}=\frac{i}{2} f(0)+\mathrm{Vp} \int_{-\infty}^{\infty} \frac{d k_{0}}{2 \pi} \frac{f\left(k_{0}\right)}{k_{0}}
$$

In this way $\delta_{2}$ divides into two parts,

$$
\delta_{2}=\delta_{2}^{\text {pole }}+\delta_{2}^{\mathrm{VP}}
$$

where $\delta_{2}^{\text {pole }}$ comes from $n^{\prime}=n$ in (42) and taking the pole part whereas $\delta_{2}^{\mathrm{VP}}$ comes from the VP-integral.

\footnotetext{
${ }^{2}$ If $\mid n>$ is not the ground state the poles in $k_{0}=E_{n}-E_{n^{\prime}}<0$ are crossed by the path and give additional contributions which we do not consider here.
} 
In $\delta_{2}^{\text {pole }}$ in this way we get rid of the $k_{0}$-integration and of the sum over $n^{\prime}$ and obtain with (43)

$$
\delta_{2}^{\text {pole }}=\frac{1}{2} \int \frac{d^{2} k_{\|}}{(2 \pi)^{2}} \frac{e^{-2 k_{\|} a}}{2 k_{\|}}\left(\frac{e^{2}}{m^{2}} \frac{1}{2} \sum_{t}|<n| p_{t}|n>|^{2}-e^{2}|<n| \varphi|n>|^{2}\right) .
$$

In taking the limes of large $a$ we expand $\varphi$ according to

$$
\begin{aligned}
& |<n| \varphi|n>|^{2}=|<n| \exp \left(i k_{t} x^{t}-k_{\|}\left(x^{3}-a\right)\right)|n>|^{2} \\
& =|<n| 1+i k_{t} x^{t}-k_{\|}\left(x^{3}-a\right)+\frac{1}{2} k_{\|}^{2}\left(x^{3}-a\right)^{2}-\frac{1}{2}\left(k_{t} x^{t}\right)^{2}+\cdots|n>|^{2} \\
& =1+\frac{1}{2} Q+\ldots
\end{aligned}
$$

and obtain

$$
\begin{aligned}
\delta_{2}^{\text {pole }} & =-\frac{e^{2}}{2 m^{2}} \int \frac{d^{2} k_{\|}}{(2 \pi)^{2}} \frac{e^{-2 k_{\|} a}}{2 k_{\|}}\left(1+k_{\|}^{2} Q+\ldots\right) \\
& =\frac{-e^{2}}{4 \pi}\left(\frac{1}{4 a}+\frac{Q}{16 a^{3}}+\ldots\right) .
\end{aligned}
$$

This pole part which involves not only the (00)-component of the new photon propagator is the contribution of $k_{0}=0$, so it is instantaneous and picks up just the electrostatic contribution.

For the considered case of an atom in front of the conducting plane we observe that the first contribution in the last line in (55) can be interpreted as the electrostatic interaction of the electron with the mirror charge of the nucleus. It does not depend on the electron state nor on a mass. Hence an identical contribution must be present if considering the same problem with electron and nucleus interchanged. Because we have to collect all distance dependent contributions to the energy we must take that contribution into account on the same footing as the first one. As a consequence we have to double the first contribution in the last line of $(55)^{3}$ and now it cancels just the electrostatic contribution of the mirror charge of the nucleus, $\delta_{\text {es,nucl }}$, Eq. (32), within the given approximation up to $O\left(\frac{1}{a^{5}}\right)$.

Now we consider the contribution of the VP-integral. After the Wick rotation with $k_{0} \rightarrow i k_{4}, \Gamma \rightarrow i \gamma \equiv i \sqrt{k_{4}^{2}+k_{\|}^{2}}$, we obtain

$$
\delta_{2}^{\mathrm{VP}}=-\mathrm{VP} \int \frac{d_{\mathrm{E}}^{3} k}{(2 \pi)^{3}} \frac{e^{-2 \gamma a}}{2 \gamma} \sum_{n^{\prime}} \sum_{s=1,2} \frac{\left.|<n| \hat{G}_{s}\left|n^{\prime}>\right|\right|^{2}}{-i k_{4}+E_{n}-E_{n^{\prime}}}
$$

\footnotetext{
${ }^{3} \mathrm{~A}$ similar contribution to $Q$ exists but it is much smaller due to the larger mass.
} 
with

$$
\begin{aligned}
\sum_{s=1,2}|<n| & \left.\hat{G}_{s}\left|n^{\prime}>\right|\right|^{2}=\frac{e^{2}}{m^{2}}\left(1+\frac{k_{4}^{2}}{\gamma^{2}}\right) \frac{1}{2} \sum_{t}|<n| p_{t}\left|n^{\prime}>\right|^{2} \\
& -e^{2} \frac{k_{\|}^{2}}{\gamma^{2}}\left[k_{\|}^{2} \frac{1}{2} \sum_{t}|<n| x_{t}\left|n^{\prime}>\right|^{2}+\gamma^{2}|<n| x^{3}-a\left|n^{\prime}>\right|^{2}\right]
\end{aligned}
$$

which follows from Eq. (43). Now we consider large $a$ and expand

$$
\frac{1}{-i k_{4}+E_{n}-E_{n^{\prime}}}=\frac{1}{E_{n}-E_{n^{\prime}}}+\frac{i k_{4}}{\left(E_{n}-E_{n^{\prime}}\right)^{2}}-\frac{k_{4}^{2}}{\left(E_{n}-E_{n^{\prime}}\right)^{3}}+\ldots .
$$

Odd powers of $k_{4}$ do not contribute for symmetry reasons. Inserting (57) and (58) into (56) we arrive at

$$
\begin{aligned}
& \delta_{2}^{\mathrm{VP}}=\int \frac{d_{\mathrm{E}}^{3} k}{(2 \pi)^{3}} \frac{e^{-2 \gamma a}}{2 \gamma} \\
& \times\left\{-\frac{e^{2}}{m^{2}}\left(1+\frac{k_{4}^{2}}{\gamma^{2}}\right) \sum_{n^{\prime}} \frac{1}{2} \sum_{t} \frac{|<n| p_{t}\left|n^{\prime}>\right|^{2}}{E_{n}-E_{n^{\prime}}}\right. \\
& +\frac{e^{2}}{m^{2}} k_{4}^{2}\left(1+\frac{k_{4}^{2}}{\gamma^{2}}\right) \sum_{n^{\prime}} \frac{1}{2} \sum_{t} \frac{|<n| p_{t}\left|n^{\prime}>\right|^{2}}{\left(E_{n}-E_{n^{\prime}}\right)^{3}} \\
& \left.+e^{2} \frac{k_{\|}^{2}}{\gamma^{2}} \sum_{n^{\prime}} \frac{k_{\|}^{2} \frac{1}{2} \sum_{t}|<n| x_{t}\left|n^{\prime}>\right|^{2}+\gamma^{2}|<n|\left(x^{3}-a\right)\left|n^{\prime}>\right|^{2}}{E_{n}-E_{n^{\prime}}}\right\} .
\end{aligned}
$$

Next we apply sum rules (74) and (175) and Eq. (76) for the static polarizabilities $\alpha_{i}$ to the sums over $n^{\prime}$. In the integration over $k$ we use spherical coordinates with $\epsilon=\cos \theta$ and obtain

$$
\begin{aligned}
\delta_{2}^{\mathrm{VP}}= & \frac{1}{4 \pi^{2}} \int_{0}^{\infty} d k k \int_{0}^{1} d \epsilon e^{-2 k a}\left\{\frac{1+\epsilon^{2}}{m}\right. \\
& \left.-k^{2}\left[\epsilon^{2}\left(1+\epsilon^{2}\right) \frac{\alpha_{1}+\alpha_{2}}{4}+\left(1-\epsilon^{2}\right)^{2} \frac{\alpha_{1}+\alpha_{2}}{4}+\left(1-\epsilon^{2}\right) \frac{\alpha_{3}}{2}\right]\right\},
\end{aligned}
$$

where the individual contributions have been written in the same order as they appear in (59). Finally we obtain

$$
\delta_{2}^{\mathrm{VP}}=\frac{e^{2}}{4 \pi} \frac{1}{6 \pi m a^{2}}-\frac{1}{32 \pi^{2} a^{4}}\left(\frac{2}{5}\left(\alpha_{1}+\alpha_{2}\right)+\frac{2}{5}\left(\alpha_{1}+\alpha_{2}\right)+\alpha_{3}\right)+O\left(\frac{1}{a^{6}}\right) .
$$

Here the first contribution cancels just $\delta_{1}$, Eq. (38), and the second one is what in the 'thin' boundary condition approach comes in place of the known CasimirPolder expression (54) for the interaction energy of an atom with a conducting 
wall,

$$
\delta_{\mathrm{CP} \text { thin }}=-\frac{1}{32 \pi^{2} a^{4}}\left(\left[\frac{\alpha_{1}+\alpha_{2}}{4}\right]+\left[\frac{11}{5} \frac{\alpha_{1}+\alpha_{2}}{4}+\alpha_{3}\right]\right) .
$$

Here the first square bracket results from the polarisation $s=1$ (it is the same as from the TE-mode in the traditional approach) and the second from $s=2$ which is different. For a spherically symmetric state with all $\alpha_{i}$ are equal we obtain

$$
\delta_{\mathrm{CP} \text { thin }}=\frac{13}{15} \delta_{\mathrm{CP} \text { thick }} \equiv \frac{13}{15} \frac{-3 \alpha}{32 \pi^{2} a^{4}},
$$

i.e., a reduction by about $13 \%$.

In the remaining part of this section we show that the result for a 'thick' conductor which in the standard approach follows with the mode expansion (14) can be obtained using a mode expansion in terms of the polarisations $E_{\mu}^{s}$, Eq. (5), with the mode functions $f_{s=0,3}\left(z, k_{3}\right)$ given by Eq. (13) instead of (9) too. In this way for 'thick' conductor boundary conditions both sets of polarisations, the traditional one, Eq. (13), and $E_{\mu}^{s}$, Eq. (5) (or the use of the photon propagator (24)) are equivalent.

The calculations follow exactly the same lines as before. In addition to the matrix elements (43) we have now

$$
\begin{aligned}
& |<n| \tilde{G}_{0}\left|n^{\prime}>\right|^{2}=e^{2} \frac{k_{0}^{2}}{\Gamma^{2}}|<n| \varphi\left|n^{\prime}>\right|^{2}+\frac{e^{2}}{m^{2}} \frac{k_{\|}^{2}}{\Gamma^{2}} \frac{1}{2} \sum_{t}|<n| p_{t}\left|n^{\prime}>\right|^{2} \\
& |<n| \tilde{G}_{3}\left|n^{\prime}>\right|^{2}=\frac{e^{2}}{m^{2}}<n\left|p_{3}\right| n^{\prime}>\left.\right|^{2}
\end{aligned}
$$

and these enter all formulas like (39) to (42) with a negative sign (cf. (24)). Then we have in addition to (47) for a single electron

$$
\begin{aligned}
& \Delta \delta_{2}^{\text {no-recoil }}=\frac{1}{i} \int \frac{d^{3} k_{\alpha}}{(2 \pi)^{3}} \frac{-e^{2 i \Gamma a}}{-2 i \Gamma} \frac{-1}{k_{0}}\left\{-e^{2} \frac{k_{0}^{2}}{\Gamma^{2}}\right. \\
& +\sum_{n^{\prime}}\left[-e^{2} \frac{k_{0}^{2}}{\Gamma^{2}}\left(k_{\|}^{2} \frac{1}{2} \sum_{t}|<n| x^{t}\left|n^{\prime}>\right|^{2}-\Gamma^{2}|<n| x^{3}-a\left|n^{\prime}>\right|^{2}\right)\right. \\
& \left.\left.-\frac{e^{2}}{m^{2}}\left(\frac{k_{\|}^{2}}{\Gamma^{2}} \frac{1}{2} \sum_{t}|<n| p_{t}\left|n^{\prime}>\right|^{2}+|<n| p_{3}\left|n^{\prime}>\right|^{2}\right)\right]\right\} .
\end{aligned}
$$

We apply the sum rules and rewrite

$$
\Delta \delta_{2}^{\text {no-recoil }}=\frac{1}{i} \int \frac{d^{3} k_{\alpha}}{(2 \pi)^{3}} \frac{-e^{2 i \Gamma a}}{-2 i \Gamma} \frac{-1}{k_{0}}\left\{-e^{2} \frac{k_{0}^{2}}{\Gamma^{2}}-\frac{e^{2}}{m^{2}} 2 \frac{k_{\|}^{2}}{\Gamma^{2}} \frac{1}{2} \sum_{t}<p_{t}^{2}>\right\} .
$$

The contributions containing $<p_{3}^{2}>$ cancelled. Carrying out the integrations we obtain finally

$$
\Delta \delta_{2}^{\text {no-recoil }}=\frac{e^{2}}{4 \pi} \frac{1}{4 a} \frac{1}{m^{2}} \sum_{t}<p_{t}^{2}>
$$


Together with $\delta_{2}^{\text {no-recoil }}$, Eq. (50) for the 'thin' conductor, these add up to the known result for the 'thick' conductor,

$$
\begin{aligned}
\delta_{2 \text { thin }}^{\text {no-recoil }}+\Delta \delta_{2}^{\text {no-recoil }} & =\delta_{2 \text { thick }}^{\text {no-recoil }} \\
& =\frac{e^{2}}{4 \pi} \frac{1}{4 a}\left(-1+\frac{1}{2 m^{2}} \sum_{t}<p_{t}^{2}>-\frac{1}{m^{2}}<p_{3}^{2}>\right) .
\end{aligned}
$$

For an atom in front of the wall we make the approximation of large $a$ and consider the additional contributions from the polarisations $s=0$ and $s=3$. It can be shown that as before the same leading contributions, i.e., that of order less than $1 / a^{4}$, cancel. The contributions to $\Delta \delta_{2}^{\mathrm{VP}}$, Eq. (156), which come in addition to (57), read

$$
\begin{aligned}
& \sum_{s=0,3} \mid<n\left|\tilde{G}_{s}\right| n^{\prime}>\left.\right|^{2} \\
&=e^{2} \frac{k_{4}^{2}}{\gamma^{2}}\left[k_{\| \mid}^{2} \frac{1}{2} \sum_{t}|<n| x_{t}\left|n^{\prime}>\right|^{2}+\gamma^{2}|<n| x^{3}-a\left|n^{\prime}>\right|^{2}\right] \\
&+\frac{e^{2}}{m^{2}}\left[\frac{2 k_{\|}^{2}}{\gamma^{2}} \frac{1}{2} \sum_{t}|<n| p_{t}\left|n^{\prime}>\right|^{2}-|<n| p_{3}\left|n^{\prime}>\right|^{2}\right] .
\end{aligned}
$$

Performing the same steps as in Eqs. (57) to (62) we find that the additional contribution is just

$$
\Delta \delta_{\mathrm{CP}}=\frac{-1}{32 \pi^{2} a^{4}} \frac{4}{10} \frac{\alpha_{1}+\alpha_{2}}{2}
$$

which together with $\delta_{\mathrm{CP}}$ thin, Eq. (62), gives the known result for the 'thick' conductor,

$$
\delta_{\mathrm{CP} \text { thin }}+\Delta \delta_{\mathrm{CP}}=\delta_{\mathrm{CP} \text { thick }}=-\frac{\alpha_{1}+\alpha_{2}+\alpha_{3}}{32 \pi^{2} a^{4}} .
$$

\section{Conclusions}

In the forging sections we have shown that the commonly known conductor boundary conditions (11) can be realized in two ways which we denote as 'thick' and 'thin' conductors. For a 'thick' conductor in addition to (11) for the normal component of the electric field on the surface the Neumann condition

$$
\frac{\partial}{\partial z} \mathcal{E}_{z}=0
$$

holds. This is the commonly used approach. It can be formulated as a mode expansion of the electromagnetic potential with the standard polarisation vectors 
as shown in Eq. (14). The boundary condition on $\mathcal{E}_{z}$ follows from considering the electric field strength belonging to (14),

$$
\begin{aligned}
\mathcal{E}_{i}= & \int d k_{\|} \int d k_{3} \frac{1}{\sqrt{2 k_{0}}} \frac{e^{i k_{\alpha} x^{\alpha}}}{2 \pi}\left\{\left(\begin{array}{c}
k_{1} \sin \left(k_{3} z\right) \\
k_{2} \sin \left(k_{3} z\right) \\
-i k_{3} \cos \left(k_{3} z\right)
\end{array}\right)_{i} \frac{-i\left(a_{0, k}-a_{3, k}\right)}{\sqrt{\pi / 2}}\right. \\
& \left.+\left(\begin{array}{c}
k_{2} \\
-k_{1} \\
0
\end{array}\right)_{i} \frac{i k_{0}}{k_{\|}} \frac{\sin \left(k_{3} z\right)}{\sqrt{\pi / 2}} a_{1, k}+\left(\begin{array}{c}
k_{1} k_{3} \sin \left(k_{3} z\right) \\
k_{2} k_{3} \sin \left(k_{3} z\right) \\
i k_{\|}^{2} \cos \left(k_{3} z\right)
\end{array}\right)_{i} \frac{i a_{2, k}}{k_{\|} \sqrt{2 \pi}}\right\} .
\end{aligned}
$$

In terms of this mode expansion there is no choice not to take $a_{0}$ in (14) going with a $\sin \left(k_{3} z\right)$ because in Coulomb gauge it is responsible for the electrostatic interaction with must fulfil a Dirichlet condition. Now, if one wishes to have $\nabla \mathcal{E}=$ 0 one needs for consistency reasons to take a $\cos \left(k_{3} z\right)$ in the third component of the polarisation which goes with $a_{3}$.

In this way, in the commonly used scheme it is natural to have the condition (172) on the normal component of the electric field. However, as demonstrated in the forgoing sections, there is a choice not to do so. There exists a set of polarisation vectors, $E_{\mu}^{s}$, Eq. (5), and a corresponding mode expansion, Eq. (4) which have the property to diagonalize the boundary conditions in the sense that only the amplitudes belonging to two polarisations ( $E_{\mu}^{s}$ with $s=1$ and $\left.s=2\right)$ are affected by the boundary conditions (II) and the other two $(s=0$ and $s=3)$ are not. The modes with $s=0,3$ do not feel the boundary at all and they are the same as without any boundary present. In this way they are defined in the whole space and penetrate the boundary freely. As a consequence we have to consider the whole space, i.e., both sides of the boundary which must be considered as infinitely thin. Accordingly, the modes with $s=1,2$ are defined separately on both sides (they do not penetrate). This setup we have called a 'thin' conductor. The existence of a consistent quantisation scheme for a 'thin' conductor had in fact been shown in [3] (even for a general, not flat surface) by the construction of the corresponding photon propagator, ${ }^{S} D_{\mu \nu}(x, y)$ and in section 2 we considered the equivalent mode expansion in detail.

In order to achieve a better understanding of the polarisations $E_{\mu}^{s}$, Eq. (5), we considered in terms of these polarisations the case of a 'thick' conductor by imposing the Neumann boundary condition (72) in addition. Now also the polarisations with $s=0,3$ become boundary dependent and the corresponding mode functions are (13) in place of (9). In terms of the propagator we have the boundary dependent part now given by E. (24) in place of (18). The equivalence to the standard mode expansion (13) is established by Eq. (15).

The difference between both types of conductors is a physical one which can be seen already on classical level. Whereas a 'thick' conductor does obviously not allow for any interaction between currents, Eq. (25), on different sides of the boundary a 'thin' conductor does in case both currents have a component in 
normal direction.

From this observation we conclude that a 'thick' conductor must assume charges which can freely move inside in perpendicular (and not only in parallel to the surface) direction. If there are no such charges, either because of the thinness of the conductor or due to an anisotropous conductivity, there is no need to impose condition (72) on $\mathcal{E}_{z}$ and this is why we call it a 'thin' conductor.

In section 3 we showed that the forces acting on a single electron or on an atom (Casimir-Polder force) in front of a conducting wall are different for both types of conductors, for instance the Casimir-Polder force is reduced by about $13 \%$ for a 'thin' conductor whereas the Casimir force between two conducting surfaces is the same.

The question whether a 'thin' conductor can be realized in experiment is beyond the scope of this paper but one may think of quasi two-dimensional systems like $C_{60}$ or other.

\section{Acknowledgements}

I thank D. Vassilevich and D. Robaschik for interesting discussions and the University of Halmstad (Sweden), where part of this work had been performed, for kind hospitality.

\section{Appendix}

Here we present the known sum rules which are used in the text:

$$
\begin{aligned}
\frac{i}{m} \frac{<n\left|p_{i}\right| n^{\prime}>}{E_{n}-E_{n^{\prime}}} & =<n\left|x_{i}\right| n^{\prime}>, \\
\sum_{n^{\prime}} \frac{|<n| p_{t}\left|n^{\prime}>\right|^{2}}{E_{n}-E_{n^{\prime}}} & =-\frac{m}{2}, \\
\frac{e^{2}}{m^{2}} \sum_{n^{\prime}} \frac{|<n| p_{i}\left|n^{\prime}>\right|^{2}}{\left(E_{n}-E_{n^{\prime}}\right)^{3}} & =-\frac{\alpha_{i}}{2} .
\end{aligned}
$$

\section{References}

[1] H.B.G. Casimir and D. Polder. The Influence of Retardation on the Londonvan der Waals Forces. Phys.Rev., 73:360-372, 1948.

[2] H.B.G. Casimir. On the attraction between perfectly conducting plates. Proc. Koninkl. Ned. Akad. Wetenshap, 51:793, 1948.

[3] M. Bordag, D. Robaschik, and E. Wieczorek. Quantum Field Theoretic Treatment of the Casimir Effect. Ann. Phys., 165:192, 1985. 
[4] M. Bordag and J. Lindig. Radiative correction to the Casimir force on a sphere. Phys. Rev., D58:045003, 1998.

[5] M. Bordag. On the Canonical Quantization of QED with Boundary Conditions. 1984. Preprint JINR-P2-84-115 (see KEK-scan).

[6] D. Robaschik and E. Wieczorek. Fluctuations of the Casimir pressure and the quantization of electrodynamics. Ann. Phys., 236:43-68, 1994.

[7] D. Robaschik and E. Wieczorek. Fluctuations of the Casimir pressure at finite temperature. Phys. Rev., D52:2341-2354, 1995.

[8] Dmitri V. Vassilevich. QED on curved background and on manifolds with boundaries: Unitarity versus covariance. Phys. Rev., D52:999, 1995.

[9] Giampiero Esposito, Alexander Yu Kamenshchik, and Giuseppe Pollifrone. Euclidean quantum gravity on manifolds with boundary. Kluwer, Dordrecht, 1997.

[10] Dmitri V. Vassilevich. The Faddeev-Popov trick in the presence of boundaries. Phys. Lett., B421:93-98, 1998.

[11] Jan Ambjorn and Richard J. Hughes. Gauge fields, BRS symmetry and the Casimir effect. Nucl. Phys., B217:336, 1983.

[12] M. Bordag, K. Kirsten, and D. V. Vassilevich. Path integral quantization of electrodynamics in dielectric media. J. Phys. A, A31:2381, 1998.

[13] M. Bordag, K. Kirsten, and D. Vassilevich. On the ground state energy for a penetrable sphere and for a dielectric ball. Phys. Rev., D59:085011, 1999.

[14] G. Barton. Quantum electrodynamics of spinless particles between conducting plates. Proc. Roy. Soc. Lond., A320:251-275, 1970.

[15] Kimball A. Milton, Jr. Lester L. DeRaad, and Julian Schwinger. Casimir selfstress on a perfectly conducting spherical shell. Ann. Phys., 115:388, 1978.

[16] E.A. Hinds. Cavity Quantum Electrodynamics. Advances in Atomic, Molecular and Optical Physics, 28:237289, 1991.

[17] Peter W. Milonni. The Quantum Vacuum: An introduction to quantum electrodynamics. Academic Press, 1993.

[18] M. Bordag. Hydrogen levels between plates. In D.Yu. Grigoriev, V.A. Matveev, V.A. Rubakov, and P.G. Tinyakov, editors, Seventh International Seminar on Quarks '92, pages 80-94. World Scientific, 1993. Preprint NTZ, University of Leipzig, 14/92. 
[19] M. Bordag. On the Apparatus Dependence of the Anomalous Magnetic Moment of the Electron. Phys. Lett., B171:113, 1986. 